\title{
Discrete Implementation of the Groves-Ledyard Mechanism
}

\author{
J. Todd Swarthout ${ }^{\dagger}$ and Mark Walker ${ }^{\ddagger}$
}

September 7, 2007

\begin{abstract}
When implementing an economic institution in the field or in the laboratory, the participants' action spaces and the institution's outcomes are typically discrete, while our theoretical analysis of the institution often assumes the sets are continuous. Predictions by the continuous model generally turn out to be good approximations to the performance of the discrete implementation. We present an example in which the continuous version has a unique and Pareto efficient equilibrium, but in which the discrete version often has vastly more equilibria, many of them far from efficient. We show that the same phenomenon appears in two experiments investigating the Groves-Ledyard mechanism, and that it may account for the experimental results.
\end{abstract}

${ }^{\dagger}$ Georgia State University Email: swarthout@gsu.edu

${ }^{\ddagger}$ University of Arizona Email: mwalker@arizona.edu

The authors are grateful for support from IBM and the International Foundation for Research in Experimental Economics (for Swarthout) and from the National Science Foundation (for Walker). 


\section{Introduction}

A common practice in economics, as in other fields, is to use continuous models to analyze phenomena that are essentially discrete. The characteristics and the predictions of the continuous model generally turn out to be good approximations to the associated discrete phenomena. It's also well understood that the differences between continuous and discrete versions of a model can sometimes matter - as in a simple Cournot model, for example, where the continuous version can have a unique equilibrium while the discrete version can have several pure strategy equilibria.

We present an example in which the usual correspondence between continuous and discrete versions of the same model fails dramatically. The example is a simple economy with a public good, in which the Groves-Ledyard mechanism (Groves and Ledyard (1977)) is used to determine how much of the public good will be provided and how much each participant will pay to finance the public good. The economy has no pathological features: consumers have quasi-linear utility functions and the public good is produced at constant marginal cost. If participants have continuous action spaces (i.e., if each participant's set of available actions is an interval in $\mathbb{R}$ ), the mechanism will have a unique equilibrium and the equilibrium will be Pareto optimal. But when participants' action spaces are discrete, we will see that the mechanism typically has multiple pure-strategy equilibria - often an enormous number of them - and there is nothing to single out any of the equilibria as focal. In further sharp contrast with the continuous model, only a small fraction of the equilibria in the discrete model may be Pareto optimal.

Under a broad class of parametrizations of the mechanism the resulting noncooperative game is supermodular; this is true whether the action spaces are continuous or discrete. When the action spaces are continuous, this supermodularity ensures that any "adaptive" behavior by the participants will converge to the unique, Pareto optimal equilibrium (Milgrom and Roberts (1990)). But when the action spaces are discrete and the set of equilibrium outcomes therefore generally quite large, supermodularity no longer guarantees efficient outcomes.

The dramatic difference between the performance of the continuous and the discrete versions of the mechanism in our example is of more than theoretical interest: in order to implement any economic mechanism - whether in an experiment or in a naturally-occurring economic setting - one has to use discrete strategy spaces. The results we present suggest that one could easily go wrong using the continuous model to make predictions about outcomes in a discrete implementation. After analyzing our example, we briefly describe two experimental studies of the Groves-Ledyard mechanism in which these issues are especially germane. 


\section{The Model}

There are two goods, which we'll call $X$ and $Y$; we denote quantities of the goods by $x$ and $y$. Good $X$ is a public good, which can be produced and provided to consumers by using $Y$ as input: it takes $c$ units of $Y$ to provide each unit of $X$. We index the $n$ participants in the economy by $i(i=1, \ldots, n)$. Each person's preference is represented by a quasi-linear utility function $u_{i}\left(x, y_{i}\right)=a_{i} x-b_{i} x^{2}-y_{i}$, where $y_{i}$ is the amount of good $Y$ person $i$ contributes to the production of $X$. It's convenient to think of $Y$ as money and $y_{i}$ as $i$ 's dollar contribution to financing the production of $X$. In order to fully cover the cost $c x$ of providing $x$ units of the public good, the values of $x$ and $y_{1}, \ldots, y_{n}$ would have to satisfy $\sum_{i} y_{i}=c x$. We assume that each person is endowed with enough of the $\mathrm{Y}$ good that the allocations we consider are all interior - i.e., no one's $y_{i}$ ever exceeds his endowment.

It is easy to show (for example, via the Samuelson condition $\sum_{i} M R S_{i}=M C$ ) that Pareto efficiency requires

$$
\text { (1) } \quad x=\frac{1}{2 B}(A-c) \quad \text { and } \quad \text { (2) } \quad \sum_{i} y_{i}=c x
$$

where $A:=\sum_{i} a_{i} \quad$ and $\quad B:=\sum_{i} b_{i}$.

We assume that the Groves-Ledyard mechanism is used to determine the levels of $x$ and $y_{1}, \ldots, y_{n}$ : each person submits a vote or message $m_{i} \in \mathbb{R}$, and then
(3) $\quad x=\sum_{i} m_{i}$
and
(4) $y_{i}=\frac{1}{n} c x+\frac{\gamma}{2}\left[\frac{n-1}{n}\left(m_{i}-\mu_{-i}\right)^{2}-\sigma_{-i}^{2}\right]$,

where $\gamma>0$ is an exogenous parameter of the mechanism, and where $\mu_{-i}$ and $\sigma_{-i}^{2}$ are defined as follows:

$$
\mu_{-i}:=\frac{1}{n-1} \sum_{j \neq i} m_{j} \quad \text { and } \quad \sigma_{-i}^{2}:=\frac{1}{n-2} \sum_{j \neq i}\left(m_{j}-\mu_{-i}\right)^{2} .
$$

Groves and Ledyard (1977; henceforth G\&L) established, for very general economies, that the Nash equilibria of this mechanism are Pareto efficient. In the economies we are considering here, with quasi-linear utility functions and a linear cost function, it is straightforward to verify that equations (3) and (4) yield equation (2), and that for any value of the parameter $\gamma$ there is a unique Nash equilibrium, which satisfies equation (1) and is therefore Pareto optimal.

While the parameter $\gamma$ has no effect on the efficiency of the equilibrium nor even (in our quasi-linear world) on the equilibrium value of $x$, it may affect the mechanism's performance 
in other ways. This has been the focus of recent research on the Groves-Ledyard mechanism, and it will be our focus here as well.

The value of $\gamma$ affects in particular the supermodularity of the mechanism (more precisely, the supermodularity of the game defined by the mechanism together with the participants' utility functions). With the quasi-linear utility functions in our example, participant $i$ 's payoff function has increasing differences if and only if ${ }^{1} \gamma>2 n b_{i}$; therefore the game is supermodular if and only if

$$
\gamma>2 n \max \left\{b_{1}, \ldots, b_{n}\right\}
$$

This necessary and sufficient condition for supermodularity holds for any message spaces that are compact subsets of $\mathbb{R}$.

Applying results in Section 3 of Milgrom and Roberts (1990; M\&R), if the mechanism has a unique equilibrium and Condition $\mathrm{S}$ is satisfied, then any adaptive dynamic process will converge to the equilibrium. Moreover, whether the equilibrium is unique or not, if the message spaces are finite then Condition S ensures that there will exist a time after which the message $n$-tuple $\left(m_{1}, \ldots, m_{n}\right)$ will always lie between the smallest and largest equilibria of the mechanism defined by $\gamma$.

\section{A Numerical Example}

Assume that the mechanism has only three participants, with utility functions

$$
u_{1}\left(x, y_{1}\right)=8 x-\frac{1}{2} x^{2}-y_{1}, \quad u_{2}\left(x, y_{2}\right)=10 x-\frac{1}{2} x^{2}-y_{2}, \quad u_{3}\left(x, y_{3}\right)=15 x-\frac{1}{2} x^{2}-y_{3}
$$

and that $c=3$ is the per-unit cost of producing the public good. We have

$$
M R S_{1}=8-x, \quad M R S_{2}=10-x, \quad M R S_{3}=15-x,
$$

and the Pareto efficient provision level of the public good is therefore $x=10$. Because

$b_{1}=b_{2}=b_{3}=\frac{1}{2}$, the game defined by the Groves-Ledayard mechanism is supermodular if and only if $\gamma>3$.

\subsection{Continuous message spaces}

Suppose that each participant, when choosing his message $m_{i}$, is allowed to choose any real number. It's straightforward to solve for the game's unique Nash equilibrium, which is

\footnotetext{
${ }^{1}$ This is shown via some straightforward but tedious algebra, contained in an appendix.
} 


$$
m_{1}=\frac{10}{3}-\frac{3}{\gamma}, \quad m_{2}=\frac{10}{3}-\frac{1}{\gamma}, \quad m_{3}=\frac{10}{3}+\frac{4}{\gamma} .
$$

The equilibrium yields the Pareto efficient public good level $x=10$ no matter what value is assigned to the parameter $\gamma$ when specifying the mechanism.

\subsection{Discrete message spaces}

Now assume that each participant's message space is discrete. In the continuous case it was straightforward to solve analytically for the equilibria and to verify that the equilibrium is always unique, but here we have to resort to computation - brute force computation. We place bounds on the discrete message spaces, to make them finite, and then we evaluate every profile of messages to determine whether any participant, by deviating to a different message, can increase his utility. The profiles from which no one has a utility-improving deviation are the Nash equilibria. With discrete message spaces, unlike the continuous case, it turns out that the number of Nash equilibria and their efficiency properties depend critically upon the details of the mechanism - the value of the mechanism's parameter $\gamma$, and the message space(s) the mechanism makes available to the participants.

Consider first the case in which the only available messages are the integers. In equation (4), the second term in the expression for $y_{i}$ imposes a "penalty" on person $i$ for choosing a message $m_{i}$ that differs from $\mu_{-i}$, the mean of the others' messages. The penalty is increasing in both $\left|m_{i}-\mu_{-i}\right|$ and $\gamma$, so it's not surprising that when $\gamma$ exceeds a certain threshold, every participant's best response (if messages are discrete) is the nearest integer to his $\mu_{-i}$. With the utility functions in our example, that threshold is approximately $\gamma=13.5$. When $\gamma$ is larger than this, the only Nash equilibria are symmetric profiles, profiles in which all three participants choose the same message. And as we increase $\gamma$, more and more of these symmetric profiles become equilibria.

The relation between $\gamma$ and the number of equilibria is described in detail in Table 1 and Figure 1. When $\gamma \leq 13.5$ there is generally a single equilibrium, which is asymmetric and at which $m_{1}+m_{2}+m_{3}=10$ : the public good is provided at the Pareto efficient level $x=10$, and the total surplus is the maximum possible, 150. For $14 \leq \gamma \leq 22$ there are two equilibria, $m_{1}=m_{2}=m_{3}=3$ and $m_{1}=m_{2}=m_{3}=4$, with corresponding (non-Pareto) provision levels $x=9$ and $x=12$. The amounts of surplus at these levels of $x$ are 148.5 and 144 , respectively. As $\gamma$ is increased, the number of equilibria grows, always with $m_{1}=m_{2}=m_{3}$, until eventually every symmetric profile is an equilibrium. (Table 1 and Figure 1 include only $0 \leq m_{i} \leq 10$, reflecting the bounds we placed on the message spaces.) Of course, as smaller and larger levels of $x$ become supportable as equilibrium outcomes, the surplus achieved at 
some of the equilibria becomes very much smaller, as can be seen in the rightmost column of Table 1.

Refining the message space eliminates neither the proliferation of equilibria nor the much lower surplus generated by many of the equilibria. If we allow each participant to use messages with one decimal place, Figure 2 shows how the number of equilibria increases with $\gamma$, and Figure 3 depicts the surplus achieved at the "worst" equilibrium for each value of $\gamma$. Note that there are again only a few rather isolated integer values of $\gamma$ at which the equilibrium is unique, and that the number of equilibria eventually increases approximately linearly with $\gamma$. However, the mechanism achieves a near-maximum level of surplus here for a broader range of $\gamma$-values than it does when using integer messages: compare Figure 3 and the rightmost column of Table 1, in which the surplus begins to fall off badly at smaller values of $\gamma$. Nevertheless, the surplus eventually falls off rather dramatically here as well, just as in the integer case.

\section{Two Experimental Papers}

Two experimental studies of the Groves-Ledyard mechanism demonstrate how these issues can arise in practice. Chen and Plott (1996; C\&P) and Chen and Tang (1998; C\&T) report on experiments in which subjects participated in the GL mechanism in economic environments that closely parallel our example.

Like our example, each experiment was an instance of the model defined above in Section 2. Each experiment consisted of several experimental sessions; in each session five subjects participated in a version of the GL mechanism (in the notation of Section 2, $n=5$ ). Each participant was assigned parameter values $a_{i}$ and $b_{i}$ as described in Table $2 .^{2}$ In C\&P the unit cost of the public good was $c=5$; in $C \& T$ it was $c=20$. Thus, the unique Pareto efficient amount of the public good in $\mathrm{C} \& \mathrm{P}$ is $x=5$ and in C\&T it is $x=25$.

In each experiment there were two kinds of sessions, or treatments: in some sessions the value of the parameter $\gamma$ was "small" $(\gamma=1)$, and in the others $\gamma$ was "large" $(\gamma=100)$. In the two "large $\gamma$ " treatments (which we denote by CP-100 and CT-100) the game defined by the mechanism and the utility functions is supermodular; in the two "small $\gamma$ " treatments (CP-1 and CT-1) the game is not supermodular.

Suppose for a moment that the participants' message spaces are continuous. Then, as in Sections 2 and 3, the GL mechanism has a unique Nash equilibrium and the amount of

\footnotetext{
${ }^{2} \mathrm{C} \& \mathrm{~T}$ explain that the parameter values and message space in their experiment were a transformation of the ones in their paper. We use the ones from the experiment.
} 
the public good at the equilibrium is the Pareto efficient amount. We refer to these as the continuous-space equilibria; they are shown in Table 3.

But of course the message spaces in the experiments were not continuous. In the C\&P experiment the message space consisted of the nine integers $-2,-1,0,1,2,3,4,5,6$; in $\mathrm{C} \& \mathrm{~T}$ it consisted of the 51 integers $-20,-19,-18, \ldots, 28,29,30$. Consequently, just as in the example presented above in Section 3, the equilibrium is unique for only some values of $\gamma$ and for other values of $\gamma$ there are many equilibria. This multiplicity of equilibria is important for interpreting the experimental results.

Those results can be described in a nutshell as follows: In treatment CT-100 the subjects' choices in each session converged quickly to the integer profile nearest to the continuousspace equilibrium in Table 3 , the profile $\left(m_{1}, m_{2}, m_{3}, m_{4}, m_{5}\right)=(5,5,5,5,5)$; the public good was therefore provided at the Pareto efficient level. In CP-100 the subjects generally chose messages relatively near the ones in Table 3, but did not coordinate on any particular profile of messages. In both CP-1 and CT-1 the subjects' choices never seemed to converge, and the public good level was generally not the Pareto amount.

If the equilibrium in each treatment were unique, a plausible explanation of these results would be the supermodularity of the $\gamma=100$ treatments and the lack of supermodularity in the $\gamma=1$ treatments.

However, the same computational approach used in Section 3 reveals that only one of the treatments, CT-100, actually has a unique equilibrium. Tables 4 and 5 describe how the set of equilibria depends upon the value of $\gamma$ in the two experiments, just as Table 1 does for our example. In particular, the treatment CT-1 has not one equilibrium, but 1,445. While all 1,445 equilibria yield the Pareto amount of the public good, we might expect subjects to have a difficult time coordinating on or converging to any particular one of them. In CP-1 there are nine equilibria, all of them again Pareto efficient, but the multiplicity might still make it difficult for subjects to arrive at any particular one of the equilibria.

Thus, the experimental subjects' failure to converge to any consistent pattern of behavior in these two "small $\gamma$ " treatments was likely a consequence of the multiplicity of equilibria and not due to the lack of supermodularity. Indeed, even if the games in these treatments were supermodular, that would guarantee only that adaptive behavior will eventually lie in the rectangle bounded by the extreme equilibria ( $c f$. Theorem 8 of Milgrom and Roberts (1990)). With so many equilibria, that would allow for an enormous range of behavior.

Behavior in the two "large $\gamma$ " treatments was more orderly, and supermodularity likely played a role. The game in treatment CT-100 is supermodular and the equilibrium is unique. 
Adaptive behavior will therefore eventually attain and remain at the equilibrium; indeed, the game is dominance solvable. Moreover, the equilibrium is a symmetric profile which might be quite focal to subjects. The rapid convergence of the subjects' observed behavior is therefore quite consistent with theoretical prediction.

The case of CP-100 is a little bit different than the other three. Here the mechanism is supermodular, like CT-100, but there are five equilibria - the five symmetric profiles $(-1,-1,-1,-1,-1),(0,0,0,0,0),(1,1,1,1,1),(2,2,2,2,2)$, and $(3,3,3,3,3)$. Theorem 8 of M\&R (see especially the theorem's second corollary) ensures that any adaptive dynamics will eventually yield only profiles in the rectangle bounded by the two extreme equilibria i.e., each participant's message $m_{i}$ will be one of the five integers $-1,0,1,2$, or 3 , but the participants will not necessarily all choose the same integer. This is consistent with the extremely limited description of the data that appears in C\&P. ${ }^{3}$

\section{$5 \quad$ Supermodularity and Multiplicity of Equilibria}

The emphasis in both $\mathrm{C} \& \mathrm{P}$ and $\mathrm{C} \& \mathrm{~T}$ is on the importance of choosing a large value for the parameter $\gamma$ in the Groves-Ledyard mechanism. C\&T's Proposition 1, they say, provides a sufficient condition for the convergence of the mechanism in quasi-linear environments, and thus gives "the precise range of $\gamma$ that induces stability under a wide class of learning dynamics."

The sufficient condition in C\&T's Proposition 1 is equivalent to our Condition S: ${ }^{4}$ if $\gamma / 2 n$ is larger than $\max \left\{b_{1}, \ldots, b_{n}\right\}$, then the mechanism is supermodular for the participants' particular utility functions. It's not the mechanism itself that's supermodular, but the game induced by the mechanism together with the participants' preferences for the public good.

Is it possible for the mechanism's designer to specify a value for the parameter $\gamma$ that will "work" for any set of participants? It might appear from Condition S that the answer is effectively yes: while we clearly can't choose a value for $\gamma$ that will work for all possible values of $b_{1}, b_{2}, \ldots, b_{n}$, we could perhaps choose a $\gamma$ so large that no plausible $b_{i}$ will exceed $\gamma / 2 n$.

\footnotetext{
${ }^{3}$ Having found explicitly all the equilibria in the two C\&P treatments, it would be extremely informative to compare the equilibria with the disaggregated raw data, especially in the supermodular treatment CP-100. At this writing, however, the authors of $\mathrm{C} \& \mathrm{P}$ have been unable to recover the raw data from the experiment.

${ }^{4}$ Proposition 1 in C\&T appeals to a result in Milgrom and Roberts (1990) that requires differentiability; it therefore applies only when message spaces are continuous (i.e., compact intervals), not to the discrete message spaces in the $\mathrm{C} \& \mathrm{P}$ and $\mathrm{C} \& \mathrm{~T}$ experiments. The direct proof we provide in an appendix applies to any compact message spaces in $\mathbb{R}$ and establishes that Condition $\mathrm{S}$ is both necessary and sufficient for supermodularity.
} 
Choosing a very large value for $\gamma$ will ensure supermodularity. But the examples in Sections 3 and 4 above suggest that if $\gamma$ is large enough to induce supermodularity for all plausible preferences, many of those preferences are likely to be ones for which the GL mechanism has multiple equilibria - perhaps many equilibria, and many of them far from efficient. Guaranteeing supermodularity is of little use when this is the case.

A positive interpretation of these results is possible, as well, however: if we know enough about the participants' preferences in advance, it may be possible to choose a value for $\gamma$ that produces both supermodularity and a unique equilibrium - and the C\&T experiment suggests that the Groves-Ledyard mechanism is likely to produce good outcomes in cases like this.

\section{Conclusion}

Economic analysis is carried out more often than not with continuous models, while implementation requires discreteness. This distinction is generally ignored because there is typically a close correspondence between the continuous and the discrete models' predictions. However, the example we've presented, and the two experiments that exhibit the same phenomenon as the example, suggest that this close correspondence can fail and the two models' predictions can differ rather dramatically. This possibility requires particular attention when uniqueness of the model's equilibrium is important. 


\section{Appendix}

For each $i=1, \ldots, n$ let $S_{i}$ be a compact subset of $\mathbb{R}$ and let $U_{i}: S \rightarrow R$, where $S=$ $S_{1} \times \ldots \times S_{n}$. This defines a normal form game $\mathbf{U}=\left(U_{1}, \ldots, U_{n}\right)$. The game $\mathbf{U}$ is said to be supermodular if each $U_{i}$ has increasing differences, to be defined below. The definition of supermodularity includes several requirements in addition to increasing differences (see Milgrom \& Roberts (1990)); each of these additional requirements is satisfied here as an immediate consequence of the compactness of each $S_{i}$ in $\mathbb{R}$ and the continuity of each $U_{i}$.

For any $m_{i}, \tilde{m}_{i} \in S_{i}$ and $\mathbf{m}_{-i}, \tilde{\mathbf{m}}_{-i} \in \times_{j \neq i} S_{j}$, let

$$
\Delta U_{i}\left(\tilde{m}_{i}, m_{i}, \mathbf{m}_{-i}\right)=U_{i}\left(\tilde{m}_{i}, \mathbf{m}_{-i}\right)-U_{i}\left(m_{i}, \mathbf{m}_{-i}\right)
$$

and

$$
\begin{aligned}
\Delta^{2} U_{i}(\tilde{\mathbf{m}}, \mathbf{m}) & =\Delta U_{i}\left(\tilde{m}_{i}, m_{i}, \tilde{\mathbf{m}}_{-i}\right)-\Delta U_{i}\left(\tilde{m}_{i}, m_{i}, \mathbf{m}_{-i}\right) \\
& =\left[U_{i}\left(\tilde{m}_{i}, \tilde{\mathbf{m}}_{-i}\right)-U_{i}\left(m_{i}, \tilde{\mathbf{m}}_{-i}\right)\right]-\left[U_{i}\left(\tilde{m}_{i}, \mathbf{m}_{-i}\right)-U_{i}\left(m_{i}, \mathbf{m}_{-i}\right)\right] .
\end{aligned}
$$

Definition: $U_{i}$ has increasing differences if $\Delta^{2} U_{i}(\tilde{\mathbf{m}}, \mathbf{m}) \geq 0$ whenever $\tilde{\mathbf{m}} \geq \mathbf{m}$.

Let $U_{i}(\mathbf{m})=v_{i}(x(\mathbf{m}))-y_{i}(\mathbf{m})$, where $x(\mathbf{m})$ and $y_{i}(\mathbf{m})$ are given by (3) and (4) in Section 2 :

$$
x(\mathbf{m})=\sum_{i} m_{i} \quad \text { and } \quad y_{i}(\mathbf{m})=\frac{1}{n} c x+\frac{\gamma}{2}\left[\frac{n-1}{n} \gamma\left[\left(m_{i}-\mu_{-i}\right)^{2}-\sigma_{-i}^{2}\right] .\right.
$$

Writing $M_{-i}$ for $\sum_{j \neq i} m_{j}$, we have

$$
U_{i}(\mathbf{m})=v_{i}\left(m_{i}+M_{-i}\right)-\frac{1}{n} c\left(m_{i}+M_{-i}\right)-\frac{n-1}{n}\left(\frac{\gamma}{2}\right)\left(m_{i}^{2}-2 \mu_{-i} m_{i}+\mu_{-i}^{2}\right)+\frac{\gamma}{2} \sigma_{-i}^{2}
$$

and therefore

$$
\begin{aligned}
\Delta U_{i}\left(\tilde{m}_{i}, m_{i}, \mathbf{m}_{-i}\right)= & {\left[v_{i}\left(\tilde{m}_{i}+M_{-i}\right)-\frac{1}{n} c\left(\tilde{m}_{i}+M_{-i}\right)\right.} \\
& \left.-\frac{n-1}{n}\left(\frac{\gamma}{2}\right)\left(\tilde{m}_{i}^{2}-2 \mu_{-i} \tilde{m}_{i}+\mu_{-i}^{2}\right)+\frac{\gamma}{2} \sigma_{-i}^{2}\right] \\
- & {\left[v_{i}\left(m_{i}+M_{-i}\right)-\frac{1}{n} c\left(m_{i}+M_{-i}\right)\right.} \\
& \left.-\frac{n-1}{n}\left(\frac{\gamma}{2}\right)\left(m_{i}^{2}-2 \mu_{-i} m_{i}+\mu_{-i}^{2}\right)+\frac{\gamma}{2} \sigma_{-i}^{2}\right] \\
= & v_{i}\left(\tilde{m}_{i}+M_{-i}\right)-v_{i}\left(m_{i}+M_{-i}\right)-\frac{1}{n} c\left(\tilde{m}_{i}-m_{i}\right) \\
& -\frac{n-1}{n}\left(\frac{\gamma}{2}\right)\left(\tilde{m}_{i}^{2}-m_{i}^{2}-2 \mu_{-i}\left(\tilde{m}_{i}-m_{i}\right)\right) .
\end{aligned}
$$


Similarly,

$$
\begin{aligned}
\Delta U_{i}\left(\tilde{m}_{i}, m_{i}, \tilde{\mathbf{m}}_{-i}\right)= & v_{i}\left(\tilde{m}_{i}+\tilde{M}_{-i}\right)-v_{i}\left(m_{i}+\tilde{M}_{-i}\right)-\frac{1}{n} c\left(\tilde{m}_{i}-m_{i}\right) \\
& -\frac{n-1}{n}\left(\frac{\gamma}{2}\right)\left(\tilde{m}_{i}^{2}-m_{i}^{2}-2 \tilde{\mu}_{-i}\left(\tilde{m}_{i}-m_{i}\right)\right)
\end{aligned}
$$

Therefore

$$
\begin{gathered}
\Delta^{2} U_{i}(\tilde{\mathbf{m}}, \mathbf{m})=v_{i}\left(\tilde{m}_{i}+\tilde{M}_{-i}\right)-v_{i}\left(m_{i}+\tilde{M}_{-i}\right)-v_{i}\left(\tilde{m}_{i}+M_{-i}\right)+v_{i}\left(m_{i}+M_{-i}\right) \\
+\frac{n-1}{n}\left(\frac{\gamma}{2}\right)\left[2 \tilde{\mu}_{-i}\left(\tilde{m}_{i}-m_{i}\right)-2 \mu_{-i}\left(\tilde{m}_{i}-m_{i}\right)\right] .
\end{gathered}
$$

The last term in the expression above can be simplified to

$$
\begin{aligned}
\frac{n-1}{n} \gamma\left(\tilde{\mu}_{-i}-\mu_{-i}\right)\left(\tilde{m}_{i}-m_{i}\right) & =\frac{n-1}{n} \gamma\left(\frac{1}{n-1} \tilde{M}_{-i}-\frac{1}{n-1} M_{-i}\right)\left(\tilde{m}_{i}-m_{i}\right) \\
& =\frac{1}{n} \gamma\left(\tilde{M}_{-i}-M_{-i}\right)\left(\tilde{m}_{i}-m_{i}\right),
\end{aligned}
$$

so that

$$
\begin{aligned}
\Delta^{2} U_{i}(\tilde{\mathbf{m}}, \mathbf{m})= & v_{i}\left(\tilde{m}_{i}+\tilde{M}_{-i}\right)-v_{i}\left(m_{i}+\tilde{M}_{-i}\right)-v_{i}\left(\tilde{m}_{i}+M_{-i}\right)+v_{i}\left(m_{i}+M_{-i}\right) \\
& +\frac{1}{n} \gamma\left(\tilde{M}_{-i}-M_{-i}\right)\left(\tilde{m}_{i}-m_{i}\right) .
\end{aligned}
$$

If $v_{i}(x)=a x-b x^{2}$ then the four $v_{i}(\cdot)$ terms in the above expression each have the form

$$
v_{i}\left(m_{i}+M_{-i}\right)=a\left(m_{i}+M_{-i}\right)-b\left(m_{i}^{2}+2 M_{-i} m_{i}+M_{-i}^{2}\right),
$$

and we have

$$
\begin{aligned}
v_{i}\left(m_{i}+M_{-i}\right)-v_{i}\left(\tilde{m}_{i}+M_{-i}\right)=a & \left(m_{i}+M_{-i}\right)-a\left(\tilde{m}_{i}+M_{-i}\right) \\
& +b\left(\tilde{m}_{i}^{2}-m_{i}^{2}+2 M_{-i} \tilde{m}_{i}-2 M_{-i} m_{i}\right) \\
=a & \left(m_{i}-\tilde{m}_{i}\right)+b\left(\tilde{m}_{i}^{2}-m_{i}^{2}\right)+2 b M_{-i}\left(\tilde{m}_{i}-m_{i}\right),
\end{aligned}
$$

and similarly

$$
v_{i}\left(\tilde{m}_{i}+\tilde{M}_{-i}\right)-v_{i}\left(m_{i}+\tilde{M}_{-i}\right)=a\left(\tilde{m}_{i}-m_{i}\right)+b\left(m_{i}^{2}-\tilde{m}_{i}^{2}\right)+2 b \tilde{M}_{-i}\left(m_{i}-\tilde{m}_{i}\right) .
$$

Therefore the four $v_{i}(\cdot)$ terms in the expression for $\Delta^{2} U_{i}(\tilde{\mathbf{m}}, \mathbf{m})$ yield

$$
\begin{aligned}
& v_{i}\left(\tilde{m}_{i}+\tilde{M}_{-i}\right)-v_{i}\left(m_{i}+\tilde{M}_{-i}\right)-v_{i}\left(\tilde{m}_{i}+M_{-i}\right)+v_{i}\left(m_{i}+M_{-i}\right)= \\
& =a\left(\tilde{m}_{i}-m_{i}\right)+a\left(m_{i}-\tilde{m}_{i}\right)+b\left(m_{i}^{2}-\tilde{m}_{i}^{2}\right)+b\left(\tilde{m}_{i}^{2}-m_{i}^{2}\right)+2 b\left(\tilde{M}_{-i}-M_{-i}\right)\left(m_{i}-\tilde{m}_{i}\right) \\
& =2 b\left(\tilde{M}_{-i}-M_{-i}\right)\left(m_{i}-\tilde{m}_{i}\right),
\end{aligned}
$$


and we have

$$
\begin{aligned}
\Delta^{2} U_{i}(\tilde{\mathbf{m}}, \mathbf{m}) & =2 b\left(\tilde{M}_{-i}-M_{-i}\right)\left(m_{i}-\tilde{m}_{i}\right)+\frac{1}{n} \gamma\left(\tilde{M}_{-i}-M_{-i}\right)\left(\tilde{m}_{i}-m_{i}\right) \\
& =\left(\frac{1}{n} \gamma-2 b\right)\left(\tilde{M}_{-i}-M_{-i}\right)\left(\tilde{m}_{i}-m_{i}\right) .
\end{aligned}
$$

The payoff function $U_{i}(\cdot)$ therefore has increasing differences if and only if $\gamma>2 n b$, and the game $\mathbf{U}=\left(U_{1}, \ldots, U_{n}\right)$ is supermodular if and only if

$$
\gamma>2 n \max \left\{b_{1}, b_{2}, \ldots, b_{n}\right\}
$$




\section{References}

[1] Chen, Yan and Charles Plott. "The Groves-Ledyard Mechansim: An Experimental Study of Institutional Design," Journal of Public Economics, 59: 335-364, (1996).

[2] Chen, Yan and FangFang Tang. "Learning and Incentive Compatible Mechanisms for Public Good Provision: An Experimental Study," Journal of Political Economy, 106: 633-662, (1998).

[3] Groves, Theodore and John Ledyard. "Optimal Allocation of Public Goods: A Solution to the 'Free Rider' Problem," Econometrica, 45: 783 - 809, (1977).

[4] Milgrom, Paul and John Roberts. "Rationalizability, Learning, and Equilibrium in Games with Strategic Complementarities," Econometrica, 58: 1255-1277 (1990). 


\begin{tabular}{|c|c|c|c|c|}
\hline Gamma & $\begin{array}{c}\text { \# of } \\
\text { Equilibria }\end{array}$ & $\begin{array}{c}\text { Common Messages } m_{i} \\
\text { in Equilibria }\end{array}$ & $\begin{array}{l}\text { Provision Levels of the } \\
\text { Public Good (x) }\end{array}$ & $\begin{array}{l}\text { Smallest } \\
\text { Surplus }\end{array}$ \\
\hline$\gamma=1$ & 3 & $\mathrm{~m}_{\mathrm{i}}$ not all the same & 10 & 150 \\
\hline $2 \leq \gamma \leq 13$ & 1 & $m_{i}$ not all the same & 10 & 150 \\
\hline $14 \leq \gamma \leq 22$ & 2 & 3,4 & 9,12 & 144 \\
\hline $23 \leq \gamma \leq 31$ & 4 & $2,3,4,5$ & $6,9,12,15$ & 112.5 \\
\hline $32 \leq \gamma \leq 40$ & 6 & $1, \ldots, 6$ & $3,6,9,12,15,18$ & 54 \\
\hline $41 \leq \gamma \leq 49$ & 8 & $0, \ldots, 7$ & $0,3,6,9,12,15,18,21$ & -31.5 \\
\hline $50 \leq \gamma \leq 58$ & 9 & $0, \ldots, 8$ & $0,3,6,9,12,15,18,21,24$ & -144 \\
\hline $59 \leq \gamma \leq 67$ & 10 & $0, \ldots, 9$ & $0,3,6,9,12,15,18,21,24,27$ & -283.5 \\
\hline $68 \leq \gamma$ & 11 & $0, \ldots, 10$ & $0,3,6,9,12,15,18,21,24,27,30$ & -450 \\
\hline
\end{tabular}

Table 1: Equilibria in the example (integer messages) 


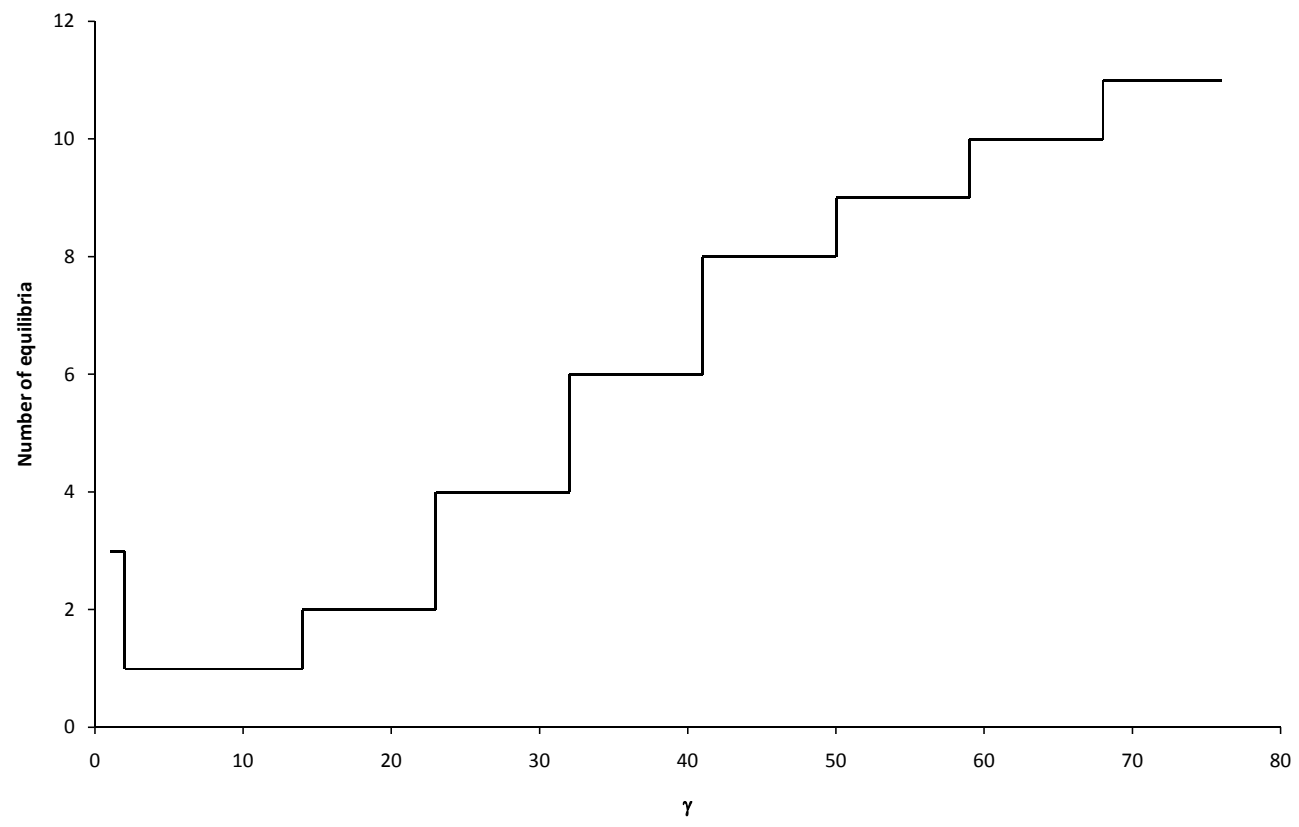

Figure 1: The number of equilibria (integer messages) 


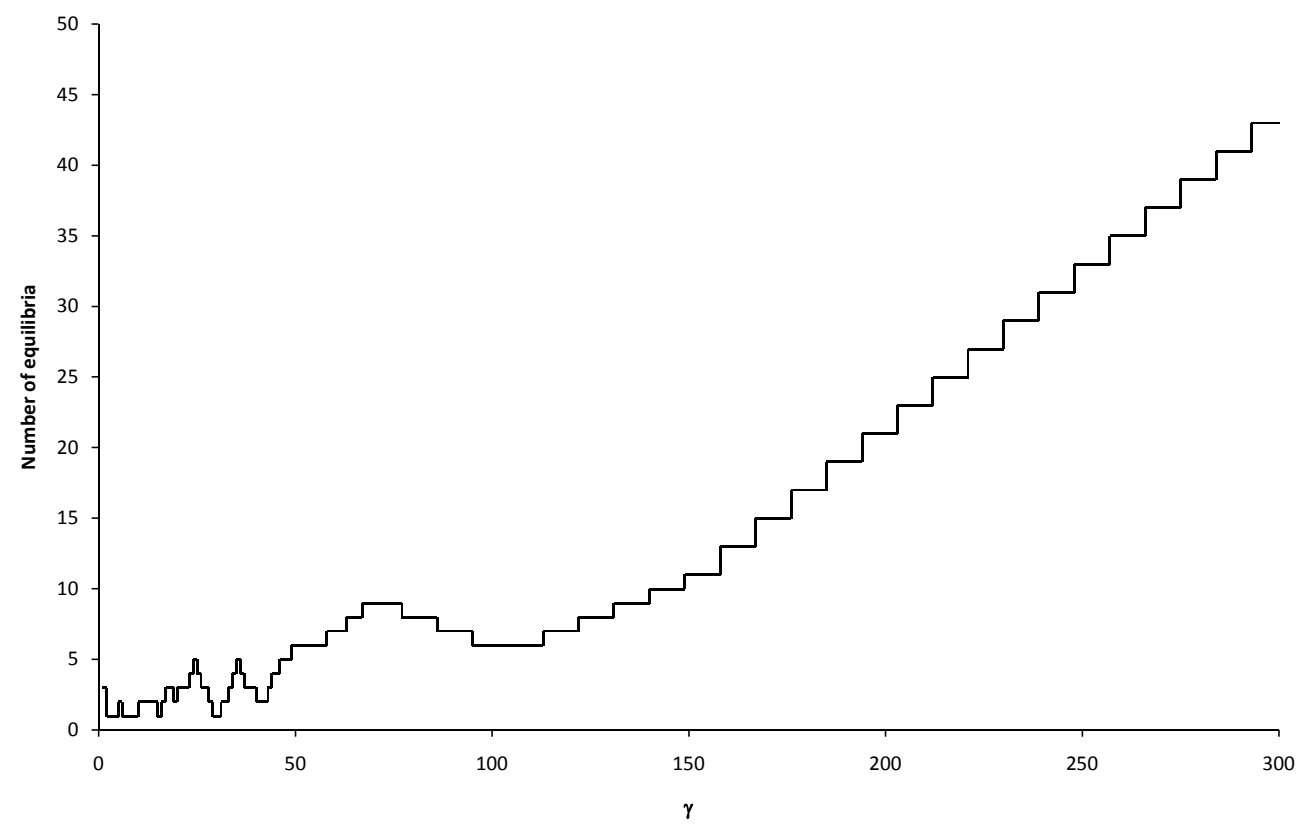

Figure 2: The number of equilibria (one-decimal-place messages) 


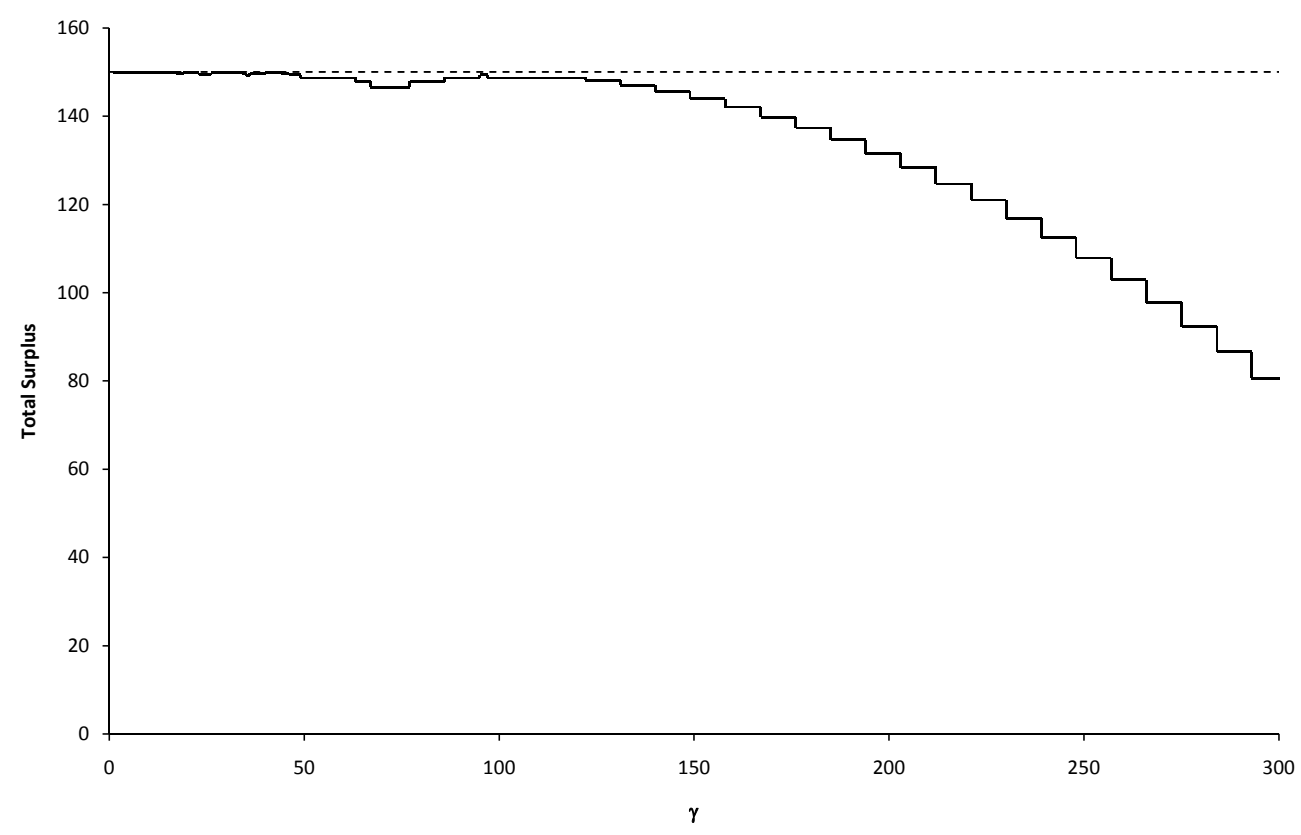

Figure 3: Surplus at the worst equilibria (one-decimal-place messages); the dashed line is at the maximum attainable surplus, 150 


\begin{tabular}{ccccccc} 
& & \multicolumn{2}{c}{ C\&P } & & \multicolumn{2}{c}{ C\&T } \\
\cline { 3 - 4 } \cline { 6 - 7 } Subject & & $a_{i}$ & $b_{i}$ & & $a_{i}$ & $b_{i}$ \\
\cline { 1 - 3 } \cline { 5 - 7 } & & -1 & 0 & & 5.2 & 0.04 \\
2 & & 5 & 0.5 & & 20.8 & 0.32 \\
3 & & 10 & 0.9 & & 7.6 & 0.08 \\
4 & & 20 & 1.8 & & 16.4 & 0.24 \\
5 & & 15 & 1.2 & & 12 & 0.16
\end{tabular}

Table 2: Preference parameters in the C\&P and C\&T experiments

\begin{tabular}{|c|c|c|c|c|c|c|c|}
\hline \multirow[b]{2}{*}{ Treatment } & \multirow[b]{2}{*}{$\gamma$} & \multicolumn{5}{|c|}{$\begin{array}{c}\text { Continuous-space } \\
\text { Equilibrium }\end{array}$} & \multirow[b]{2}{*}{$x$} \\
\hline & & $\mathrm{m} 1$ & $\mathrm{~m} 2$ & m3 & $\mathrm{m} 4$ & $\mathrm{m5}$ & \\
\hline $\mathrm{CP}-1$ & 1 & -1 & 0 & 1 & 2 & 3 & 5 \\
\hline CP-100 & 100 & .98 & .99 & 1 & 1.01 & 1.02 & 5 \\
\hline CT-1 & 1 & -15 & 25 & -5 & 15 & 5 & 25 \\
\hline CT-100 & 100 & 4.8 & 5.2 & 4.9 & 5.1 & 5 & 25 \\
\hline
\end{tabular}

Table 3: Continuous-space equilibria in the C\&P and C\&T experiments 


\begin{tabular}{|c|c|c|c|c|}
\hline$\gamma$ & \#NE & $\begin{array}{c}\text { Common messages } \\
m_{\mathrm{i}} \text { in NE }\end{array}$ & Provision levels of the public good & smallest surplus \\
\hline 1 & 9 & $m_{i}$ not all the same & 5 & 110 \\
\hline 2 & 4 & $m_{i}$ not all the same & 5 & 110 \\
\hline 3 & 2 & $m_{i}$ not all the same & 5 & 110 \\
\hline 4 & 0 & & & \\
\hline $5 \leq \gamma \leq 31$ & 1 & 1 & 5 & 110 \\
\hline $32 \leq \gamma \leq 38$ & 2 & $m_{i}$ not all the same & 5,9 & 39.6 \\
\hline $39 \leq \gamma \leq 43$ & 2 & 1,2 & 5,10 & 0 \\
\hline $44 \leq \gamma \leq 83$ & 3 & $0,1,2$ & 0,510 & 0 \\
\hline $84 \leq \gamma \leq 87$ & 4 & $0,1,2,3$ & $0,5,10,15$ & -330 \\
\hline $88 \leq \gamma \leq 127$ & 5 & $-1, \ldots, 3$ & $-5,0,5,10,15$ & -330 \\
\hline $128 \leq \gamma \leq 132$ & 6 & $-1, \ldots, 4$ & $-5,0,5,10,15,20$ & -880 \\
\hline $133 \leq \gamma \leq 172$ & 7 & $-2, \ldots, 4$ & $-10,-5,0,5,10,15,20$ & -880 \\
\hline $173 \leq \gamma \leq 217$ & 8 & $-2, \ldots, 5$ & $-10,-5,0,5,10,15,20,25$ & -1650 \\
\hline $218 \leq \gamma$ & 9 & $-2, \ldots, 6$ & $-10,-5,0,5,10,15,20,25,30$ & -2640 \\
\hline
\end{tabular}

Table 4: Equilibria in the C\&P experiment 


\begin{tabular}{|c|c|c|c|c|}
\hline$\gamma$ & \# NE & $\begin{array}{c}\text { Common } \\
\text { messages } \\
\mathrm{m}_{\mathrm{i}} \text { in } \mathrm{NE}\end{array}$ & $\begin{array}{l}\text { Provision } \\
\text { levels of the } \\
\text { public good }\end{array}$ & $\begin{array}{c}\text { Smallest } \\
\text { surplus }\end{array}$ \\
\hline 1 & 1445 & $\mathrm{~m}_{\mathrm{i}}$ not all the same & 25 & 525 \\
\hline 2 & 97 & $m_{i}$ not all the same & 25 & 525 \\
\hline 3 & 54 & $m_{i}$ not all the same & 25 & 525 \\
\hline 4 & 22 & $m_{i}$ not all the same & 25 & 525 \\
\hline 5 & 9 & $\mathrm{~m}_{\mathrm{i}}$ not all the same & 25 & 525 \\
\hline 6 & 14 & $m_{i}$ not all the same & 25 & 525 \\
\hline 7 & 4 & $m_{i}$ not all the same & 25 & 525 \\
\hline 8 & 3 & $m_{i}$ not all the same & 25 & 525 \\
\hline 9 & 2 & $\mathrm{~m}_{\mathrm{i}}$ not all the same & 25 & 525 \\
\hline 10 & 3 & $m_{i}$ not all the same & 25 & 525 \\
\hline $11 \leq \gamma \leq 13$ & 1 & $m_{i}$ not all the same & 25 & 525 \\
\hline $14 \leq \gamma \leq 17$ & 2 & $m_{i}$ not all the same & 25 & 525 \\
\hline $18 \leq \gamma \leq 35$ & 1 & $\mathrm{~m}_{\mathrm{i}}$ not all the same & 25 & 525 \\
\hline $36 \leq \gamma \leq 46$ & 0 & & & \\
\hline $47 \leq \gamma \leq 129$ & 1 & 5 & 25 & 525 \\
\hline $130 \leq \gamma \leq 229$ & 2 & 5,6 & 25,30 & 504 \\
\hline $230 \leq \gamma \leq 329$ & 3 & $4,5,6$ & $20,25,30$ & 504 \\
\hline $330 \leq \gamma \leq 429$ & 4 & $4,5,6,7$ & $20,25,30,35$ & 441 \\
\hline $430 \leq \gamma \leq 529$ & 5 & $3,4, \ldots, 7$ & $15,20, \ldots, 35$ & 441 \\
\hline $530 \leq \gamma \leq 629$ & 6 & $3,4, \ldots, 8$ & $15,20, \ldots, 40$ & 336 \\
\hline $630 \leq \gamma \leq 730$ & 7 & $2,3, \ldots, 8$ & $10,15, \ldots, 40$ & 336 \\
\hline $731 \leq \gamma \leq 829$ & 8 & $2,3, \ldots, 9$ & $10,15, \ldots, 45$ & 189 \\
\hline $830 \leq \gamma \leq 929$ & 9 & $1,2, \ldots, 9$ & $5,10, \ldots, 45$ & 189 \\
\hline $930 \leq \gamma \leq 1029$ & 10 & $1,2, \ldots, 10$ & $5,10, \ldots, 50$ & 0 \\
\hline $1030 \leq \gamma \leq 1129$ & 11 & $0,1, \ldots, 10$ & $0, \quad 5, \ldots, 50$ & 0 \\
\hline $1130 \leq \gamma \leq 1229$ & 12 & $0,1, \ldots, 11$ & $5, \ldots, 55$ & -231 \\
\hline $1230 \leq \gamma \leq 1330$ & 13 & $-1, \quad 0, \ldots, 11$ & $0, \ldots, 55$ & -231 \\
\hline $1331 \leq \gamma \leq 1429$ & 14 & $-1, \quad 0, \ldots, 12$ & $-5, \quad 0, \ldots, 60$ & -504 \\
\hline $1430 \leq \gamma \leq 1529$ & 15 & $-2,-1, \ldots, 12$ & $-10,-5, \ldots, 60$ & -504 \\
\hline $1530 \leq \gamma \leq 1630$ & 16 & $-2,-1, \ldots, 13$ & $-10,-5, \ldots, 65$ & -819 \\
\hline $1631 \leq \gamma \leq 1729$ & 17 & $-3,-2, \ldots, 13$ & $-15,-10, \ldots, 65$ & -819 \\
\hline $1730 \leq \gamma \leq 1830$ & 18 & $-3,-2, \ldots, 14$ & $-15,-10, \ldots, 70$ & -1176 \\
\hline $1831 \leq \gamma \leq 1929$ & 19 & $-4,-3, \ldots, 14$ & $-20,-15, \ldots, 70$ & -1176 \\
\hline $1930 \leq \gamma \leq 2030$ & 20 & $-4,-3, \ldots, 15$ & $-20,-15, \ldots, 75$ & -1575 \\
\hline $2031 \leq \gamma \leq 2129$ & 21 & $-5,-4, \ldots, 15$ & $-25,-20, \ldots, 75$ & -1575 \\
\hline $2130 \leq \gamma \leq 2230$ & 22 & $-5,-4, \ldots, 16$ & $-25,-20, \ldots, 80$ & -2016 \\
\hline $2231 \leq \gamma \leq 2330$ & 23 & $-6,-5, \ldots, 16$ & $-30,-25, \ldots, 80$ & -2016 \\
\hline $2331 \leq \gamma \leq 2430$ & 24 & $-6,-5, \ldots, 17$ & $-30,-25, \ldots, 85$ & -2499 \\
\hline $2431 \leq \gamma \leq 2530$ & 25 & $-7,-6, \ldots, 17$ & $-35,-30, \ldots, 85$ & -2499 \\
\hline $2531 \leq \gamma \leq 2630$ & 26 & $-7,-6, \ldots, 18$ & $-35,-30, \ldots, 90$ & -3024 \\
\hline $2631 \leq \gamma \leq 2729$ & 27 & $-8,-7, \ldots, 18$ & $-40,-35, \ldots, 90$ & -3024 \\
\hline $2730 \leq \gamma \leq 2829$ & 28 & $-8,-7, \ldots, 19$ & $-40,-35, \ldots, 95$ & -3591 \\
\hline $2830 \leq \gamma \leq 2930$ & 29 & $-9, \quad-8, \ldots, \quad 19$ & $-45,-40, \ldots, 95$ & -3591 \\
\hline $2931 \leq \gamma \leq 3029$ & 30 & $-9,-8, \ldots, 20$ & $-45,-40, \ldots, 100$ & -4200 \\
\hline $3030 \leq \gamma \leq 3130$ & 31 & $-10,-9, \ldots, 20$ & $-50,-45, \ldots, 100$ & -4200 \\
\hline $3131 \leq \gamma \leq 3230$ & 32 & $-10,-9, \ldots, 21$ & $-50,-45, \ldots, 105$ & -4851 \\
\hline $3231 \leq \gamma \leq 3329$ & 33 & $-11,-10, \ldots, 21$ & $-55,-50, \ldots, 105$ & -4851 \\
\hline $3330 \leq \gamma \leq 3430$ & 34 & $-11,-10, \ldots, 22$ & $-55,-50, \ldots, 110$ & -5544 \\
\hline $3431 \leq \gamma \leq 3530$ & 35 & $-12,-11, \ldots, \quad 22$ & $-60,-55, \ldots, 110$ & -5544 \\
\hline $3531 \leq \gamma \leq 3629$ & 36 & $-12,-11, \ldots, 23$ & $-60,-55, \ldots, 115$ & -6279 \\
\hline $3630 \leq \gamma \leq 3729$ & 37 & $-13,-12, \ldots, 23$ & $-65,-60, \ldots, 115$ & -6279 \\
\hline $3730 \leq \gamma \leq 3829$ & 38 & $-13,-12, \ldots, 24$ & $-65,-60, \ldots, 120$ & -7056 \\
\hline $3830 \leq \gamma \leq 3930$ & 39 & $-14,-13, \ldots, 24$ & $-70,-65, \ldots, 120$ & -7056 \\
\hline $3931 \leq \gamma \leq 4029$ & 40 & $-14,-13, \ldots, 25$ & $-70,-65, \ldots, 125$ & -7875 \\
\hline $4030 \leq \gamma \leq 4129$ & 41 & $-15,-14, \ldots, 25$ & $-75,-70, \ldots, 125$ & -7875 \\
\hline $4130 \leq \gamma \leq 4230$ & 42 & $-15,-14, \ldots, 26$ & $-75,-70, \ldots, 130$ & -8736 \\
\hline $4231 \leq \gamma \leq 4329$ & 43 & $-16,-15, \ldots, 26$ & $-80,-75, \ldots, 130$ & -8736 \\
\hline $4330 \leq \gamma \leq 4430$ & 44 & $-16,-15, \ldots, 27$ & $-80,-75, \ldots, 135$ & -9639 \\
\hline $4431 \leq \gamma \leq 4530$ & 45 & $-17,-16, \ldots, 27$ & $-85,-80, \ldots, 135$ & -9639 \\
\hline $4531 \leq \gamma \leq 4629$ & 46 & $-17,-16, \ldots, 28$ & $-85,-80, \ldots, 140$ & -10584 \\
\hline $4630 \leq \gamma \leq 4729$ & 47 & $-18,-17, \ldots, 28$ & $-90,-85, \ldots, 140$ & -10584 \\
\hline $4730 \leq \gamma \leq 4830$ & 48 & $-18,-17, \ldots, 29$ & $-90,-85, \ldots, 145$ & -11571 \\
\hline $4831 \leq \gamma \leq 4929$ & 49 & $-19,-18, \ldots, 29$ & $-95,-90, \ldots, 145$ & -11571 \\
\hline $4930 \leq \gamma \leq 5030$ & 50 & $-19,-18, \ldots, 30$ & $-95,-90, \ldots, 150$ & -12600 \\
\hline $5031 \leq \gamma$ & 51 & $-20,-19, \ldots, 30$ & $-100,-95, \ldots, 150$ & -12600 \\
\hline
\end{tabular}

Table 5: Equilibria in the C\&T experiment 